\title{
Olivier Rolin, écrivain sans contrée
}

\author{
Olivier Rolin - the Writer without a Country
}

\begin{abstract}
RÉSUMÉ
L'auteur Olivier Rolin est un écrivain bourlingueur qui depuis un demi-siècle aime partager ses pérégrinations. Il utilise ses carnets de voyage pour recomposer des paysages sensoriels basés sur une entreprise d'écriture de l'errance fondée sur une carte qui combine voyage et littérature. L'écrivain propose une géographie personnelle et s'interroge sur son attrait pour les ruines de paysages dans des régions traversées. Il esquisse ainsi une trajectoire autobiographique et travaille à se dépendre de lui-même à travers une féconde extériorité.

Mots clés : bourlingueur, mémoire, extérieur, mosaïque, ruines, Olivier Rolin
\end{abstract}

\section{ABSTRACT}

The writer Olivier Rolin has been working as a globetrotting writer for half a century and likes to share his peregrinations. He uses his travel diaries to recompose sensorial landscapes based on an enterprise of wandering writing and on a map that combines travel and literature. The writer proposes a personal geography and wonders about his attraction to the ruins of landscapes in no go zones. He thus sketches an autobiographical trajectory and works to depend on himself through a fruitful externality.

Keywords: bourlingueur, memory, exteriority, mosaic, ruin, Olivier Rolin

L'écrivain Olivier Rolin poursuit un travail autobiographique qui ne dit pas son nom. En effet, cet auteur, depuis les années quatre-vingts, propose une littérature qui se nourrit expressément de ses voyages autour du monde. Elle dessine en creux le parcours effectif d'un exceptionnel bourlingueur et une trajectoire de vie qui s'élabore par les notes compilées dans des carnets de bord. Ainsi, l'auteur dans un dernier ouvrage intitulé Extérieur monde, publié en 2019 poursuit la constitution d'une œuvre de la géo-graphie de soi. Il s'annonce également comme un testament de recomposition d'une existence, propose un parcours géographique par les bribes de voyages narrées et une pérégrination littéraire jubilatoire par les convocations des auteurs qui ont accompagné cet Ulysse moderne, désormais rentré au pays du bilan. L'auteur allie espaces littéraires et étendues du monde qu'il va parcourir de la Russie en passant par le Moyen-Orient, l'Asie et l'Europe jusqu'à l'Amérique du Sud...

Antoine Jurga, Université de Valenciennes et du Hainaut-Cambrésis, Campus du Mont-Houy, Le Mont Houy 59313 Valenciennes cedex 9, antoine.jurga@free.fr, http://orcid.org/0000-0001-9928-8558 
Rolin choisit dans Extérieur monde de progresser par une constante digression qui permet d'associer les êtres et les paysages, les souvenirs et les déplacements, les lieux et les présences, d'avancer par une errance propre à dessiner un parcours d'homme libre. Il propose une «façon éclatée et inversée, partant en quelque sorte de l'extérieur », (Rolin, 2019, p. 70) selon l'expression de l'auteur, de restituer ses vagabondages et déambulations. Le décentrement volontaire de l'écrivain baroudeur permet ainsi d'éviter le classique récit de voyage ou encore les traditionnels mémoires. A l'inverse, il autorise l'élaboration d'une cartographie littéraire que chacun peut traverser en lisant le dernier ouvrage ou encore en redécouvrant l'œuvre entière depuis cette dernière publication. L'ouvrage Extérieur monde compose une proposition authentique qui, par l'assemblage de morceaux de vie soigneusement consignés durant des années, parvient à reconstituer l'homme dans une vérité énonçant ses zones de troubles et d'inénarrable. Sa littérature élabore de cette manière une mémoire des lieux et des individus où se construisent des mythes spatiaux personnels que dessine la traversée de l'existence. Ce récit fragmentaire veut se tenir à la hauteur d'un labeur scriptural de consignation immédiat traversant le $\mathrm{XX}^{\mathrm{e}}$ siècle pour élaborer un récit de soi a posteriori et offrir un périple savant dans les textes des grands auteurs. Comment l'écrivain-bourlingueur, dans cet ouvrage au titre très révélateur de sa démarche, Extérieur monde, parvient-il à élaborer une géo-auto-graphie de son existence fondée sur la mémoire des lieux?

Rolin propose depuis ses premiers ouvrages une littérature déconcertante qui cherche à produire un certain inconfort chez ses lecteurs propre à susciter des émotions et des constructions d'images que l'écriture aura déclenchées. Ses récits, qui s'appuient sur des pérégrinations effectives, bénéficient d'un plan réflexif sur son art d'écrire et des publications fondées sur une réflexion théorique de son propre travail. Cet aspect fut formalisé grâce à des conférences qui ont été rassemblées par exemple dans l'ouvrage Bric et broc. Donc, il faut stipuler que Rolin a une pleine conscience de sa littérature, de sa visée et des effets recherchés. En effet, l'auteur livre les clefs de sa démarche pour la restitution de ses voyages sous la forme de récits qui s'éloignent pour la plupart d'une narration classique dont il a conscience : « Je sais bien qu'on ne doit pas commencer un livre comme ça » (Rolin, 2019, p. 11). L'inconfort fructueux, que Rolin propose, repose sur des récits fragmentaires qui privilégient les réminiscences à travers un assemblage à l'aune du volume publié et de l'ensemble des publications qui augmente, régulièrement depuis 1983, son territoire littéraire.

Dans Extérieur monde, Rolin évoque le décentrement essentiel pour se dire. Le mouvement se produit donc de l'extérieur vers l'individu, l'écrivain refuse le principe narratologique des mémoires (« Pas des mémoires, grand Dieu non!», Rolin, 2019, p. 11) et évite soigneusement les épanchements intimes, à l'exception de quelques passages par exemple avec l'actrice Jane Birkin, qui fut sa compagne ; 
il privilégie une perception qui en passe avant tout par une expérience du monde, à l'instar d'un de ses maîtres à penser, Blaise Cendrars. L'épigone Rolin se considère humblement comme une sorte de réceptacle des images du monde, des lieux traversés, des êtres rencontrés, des paysages observés : il accepte d'être un écran sur lequel s'impriment des images des géographies variées. Il explique, à propos des diverses rencontres en divers lieux, dans les dernières lignes de Extérieur monde que : « Chacun a déposé en moi quelque chose que je ne saurais pas nommer [...] une très mince pellicule, de savoir, d'émotion, de rêve, et toutes ensemble ont composé à la fin ma vieille écaille jaspée de tortue marine » (p. 298). Il est certain que Rolin conserve depuis ses études un intérêt marqué pour l'Histoire et en particulier l'Antiquité ${ }^{1}$, il se veut celui qui observe les mondes présents ou enfouis à travers les ruines et les traces de l'ancien. Mais il se veut également géographe ; celui qui décrit les « événements » à la surface de la terre. L'antique écaille de la tortue, à laquelle se compare l'auteur, figure une enveloppe sur laquelle un palimpseste du monde s'écrit depuis les origines du vivant à l'aide de trois apports essentiels « de savoir, d'émotion, de rêve ». Les individus rencontrés au cours des périples ont laissé une trace certaine que l'écrivain conserve en lui (de nombreux paragraphes de Extérieur monde pp. 239 à 272 sont consacrés aux femmes croisées, l'ouvrage Bar des flots noirs est constitué de portraits de femmes dont il restitue la mémoire) mais la rencontre avec les paysages urbains ou ruraux des plus variés à travers la planète constitue également une part de cette écriture palimpsestique à laquelle le corps de l'écrivain ne fait que participer en qualité de relais d'une parole commencée bien avant lui. Il précise au début du récit Extérieur monde dans un commentaire parembolique l'importance des traces que l'écrivain peut enregistrer en scrutant le monde avec attention : « je prétends être un écrivain moderne [...]. Pas des mémoires, donc, mais peut-être le relevé des traces que le monde laisse sur une vie - ou plutôt, des traces dont le monde compose le tableau d'une vie » (p. 12). Il stipule ainsi dans ce commentaire le sens inversé de la lecture habituelle qui fait de l'homme un observateur volontaire : il s'agit plutôt de relever des traces que « le monde laisse sur une vie ", en l'occurrence celle de l'écrivain. Il aime à évoquer le travail de l'écrivain qui produit une sorte de fresque en composant un ensemble de « tableaux » des contrées, des villes, des montagnes, des êtres... L'auteur invite donc le lecteur à penser l'existence comme une élaboration a posteriori et intentionnelle d'une représentation de soi dont la conscience tient avant tout à une construction iconique intérieure qui cumule des paysages, instants, individus, situations... et que le récit peut reconstruire sous une traduction scripturale propre à susciter des déflagrations d'images².

1 « il faut ajouter le latin et le grec, par lesquels je crois j'en suis venu à l'amour de ma langue » (Rolin, 2019, p. 13).

2 «l'immédiate, délicieuse et totale déflagration du souvenir »(Proust, 1925, p. 327). 
Dans les premières pages de Extérieur monde, l'auteur évoque le travail minutieux d'un ramendeur de poterie au service d'archéologues en Egypte pour définir son propre travail. En effet, nous pouvons élaborer un parallèle car l'image est particulièrement bien choisie. Le ramendeur et l'écrivain ont un intérêt certain pour les objets de l'Antiquité, une capacité à l'observation des bribes que les mondes enfouis donnent à lire, une patience et une fulgurance de la recomposition, un goût marqué pour l'assemblage de fragments, un intérêt pour les jonctions visibles de la restauration et la résurgence de ce qui fut. Le ramendeur propose la recréation d'une poterie ancienne, l'écrivain élabore un livre et tous deux témoignent d'un passé, d'un vécu, d'une présence de l'homme et de son génie. La même humilité est perceptible dans les deux approches ; celle du rabouteur au service du recollage des morceaux et celle de l'écrivain comparé à un modeste réservoir des souvenirs du monde. Seul l'homme attentif aux mouvements du monde et aux strates de l'Histoire peut les dire. Rolin choisit le temps verbal du futur de l'indicatif pour le dernier verbe « serai » qui permet de confirmer assurément ce statut, articulé avec une négation qui exprime une réduction de l'homme à un vide central. Cette image rejoint celle de l'écaille de vieille tortue ; l'enveloppe recèle les images du monde sous forme de souvenirs tandis que l'écrivain gagne un statut de vacance propre à une disposition de spectateur érudit et avisé.

Une autre image à l'esprit : celle d'un jeune ramendeur de poteries, en Égypte, à Saqqara. On disposait devant lui, sur le sable, les dizaines de tessons remontés de la fouille dans des bannes de tiges de palmiers. Il réfléchissait longuement, silencieux, immobile, assis en tailleur comme les scribes et les serviteurs royaux dont les silhouettes peintes se voyaient encore sur les blocs de calcaire à une dizaine de mètres sous la surface, au fond du puits de fouille. Puis, sans apparente hésitation, il choisissait trois ou quatre tessons, les encollait, les assemblait, et les cassures en effet s'ajustaient. Puis il se replongeait dans sa contemplation immobile, avant de recommencer. À la fin de la journée, il pouvait avoir reconstitué un vase canope. [...] C'est le même genre de travail que j'entreprends : rabouter, coller des dizaines d'éclats de souvenirs, en recomposer un vase imparfait, fracturé, dont je ne serai que le vide central (Rolin, 2019, p. 43).

Rolin dans Extérieur monde recompose les divers lieux convoqués par sa mémoire et les notes conservées dans ses carnets depuis des années comme une suite de divers tableaux et montre les marques de cette composition qui se veut visuelle, telle une mosaïque qui laisse visibles les sutures qui lient l'assemblage des éclats. La lecture, selon Rolin, exige un certain recul, un regard distancié pour recevoir l'image d'ensemble faisant abstraction des jonctions. Ces dernières entre les « morceaux » du livre fonctionnent comme celles d'une poterie antique reconstituée qui conserve les marques du travail de juxtaposition et d'élaboration de collusions d'images fragmentaires du monde. En effet, sans se soucier d'une quelconque chronologie ou tension narrative, l'auteur élabore sa marqueterie 
personnelle ${ }^{3}$. En tournant la page, le lecteur change de lieu sans transition car il s'agit pour Rolin de façonner un récit composé de fragments. Sont évoqués des lieux les plus divers sans hiérarchie, ni ordre ni enchaînement comme un recueil de je me souviens... qui apporte une grande vérité à l'ensemble : « Il y a deux types, deux jeunes, à Shangaï » (p. 175), « Je traverse Central Park » (p. 184), «Pour Moscou » (p. 189), «Je prends le bus à Khartoum pour Port-Soudan » (p. 202), " De même, à Shangaï » (p. 214), "A Mexico j'avais rencontré le traducteur » (p. 220), etc. Cette énumération de relevés, loin d'être exhaustive, poursuit sa déclinaison à travers les différents ouvrages publiés depuis 1983 ; l'un évoque les îles Solovki, l'autre Port-Soudan, l'autre encore Méroé, Veracruz, le lac Baïkal... L'auteur sait que les ouvrages, ainsi constitués en un ensemble, permettent de s'approcher d'une forme de vérité qui tente de s'ériger par une proposition de pleins et de liants qui «fabriquent» le bel œuvre et qui constitue également un reflet du rapport de l'auteur au monde et au livre dans une proclamation d'équivalence. Ainsi, l'auteur aime à reconstituer pour le lecteur des assemblages d'images comme une mosaïque qui propose des descriptions des lieux pour figurer une effective cartographie du monde dessinée par les voyages et la mémoire de l'écrivain. Par exemple, dans Extérieur monde, l'auteur livre ses émotions face à certains paysages notamment en Afghanistan lors de sa rencontre avec le général Massoud. A travers la description, Rolin précise en quoi le paysage peut proprement s'imposer à l'observateur et produire une sidération pour laquelle l'auteur évoque le satori d'un autre bourlingueur, Jack Kerouac. Selon un parallèle constant, l'auteur associe cette impression à celle produite par la lecture, " certaines pages invitent à suspendre la lecture, à les lire dans le silence » (p. 285), qui véritablement subjugue. L'écrivain voyageur est avant tout un observateur des images produites par les paysages et les rencontres ; il n'en est que le scribe.

En contrebas, entre les lignes, s'étendait un lac ou un marais gelé, sur lequel on entendait couiner des canards partisans de la neutralité. Tout, ce jour-là, était extraordinairement calme, le ciel gris immobile, le front. Si l'on ne regardait que le lac gelé et les canards, on pouvait se croire dans un tableau hollandais. Mais en haut, entre les murs de la forteresse déjà à demi détruite au dixneuvième siècle par les guerres anglo-afghanes, c'était un cimetière de tanks. Un T-55 dont le canon éclaté avait l'apparence rebroussée d'une peau de banane menaçait les canards, un autre, déchiqueté par des roquettes d'avion, se dressait en surplomb, comme s'apprêtant à plonger, audessus des quartiers sud de la ville estompés par une brume mauve, sa tourelle éjectée à cinq mètres, canon planté en terre. Un chat miaulait dans l'épave d'un blindé russe à huit roues. Un transistor posé sur un char diffusait une musique afghane aigrelette. Sous les murailles, la piscine des rois Nadir et Zaher était bien amochée, on n'y nagerait plus avant longtemps. Les tours plus que médiévales, les monstres mécaniques d'acier éclaté, la neige et la glace, les

3 « ce n'est qu'une marqueterie mal jointe » de Montaigne (1963/1588, pp. 941-942). « Je fais énormément de marqueterie quand j'écris, et quelquefois j'en fais trop » (Bouvier, 1997, p. 152). 
nuages, le chat, la musique, les montagnes, la ville en contrebas presque effacée : dans le genre « paysage de guerre », la composition était parfaite [guillemets de l'auteur cité] (pp. 286-287).

Ici, Rolin décrit un paysage désolé qui témoigne de la violence des combats et dans lequel se croisent des éléments manufacturés pour la guerre et d'autres surgis de la nature tels les animaux, le climat et les reliefs de la région. Double mouvement qui induit le retour à des temps plus sereins après les luttes armées mais qui s'appuie également sur une articulation entre ruines de la guerre et renouveau par des éléments réduits mais certains : « un chat miaulait dans l'épave » qui, in fine, engendrent des « ruines de texte». Rolin met en exergue les temporalités qu'il fait entrer en collusion par superposition des époques, celle des combats contre l'armée russe, celle du voyage de l'écrivain, celle des conflits du $\mathrm{XIX}^{\mathrm{e}}$ siècle, celle plus ancienne des temps médiévaux. Il conclut le paragraphe par une formulation qui indique à la fois la séduction produite par le paysage qui instaure l'état de perfection et le recul de l'écrivain qui apprécie la composition produite par le hasard mais qu'il envisage par le filtre de la représentation visuelle (se croire dans un tableau hollandais) ou scripturale par la reproduction qu'il livre.

Le paragraphe qui propose cette description comme une rencontre extraordinaire avec un paysage est particulièrement balisé pour en permettre une reconstitution des plus précises et fidèles pour le lecteur. En effet, l'auteur recourt à des indices spatiaux nombreux d'une géographie du proche. Ainsi, il alerte le lecteur sur la nécessité de produire un cadre pour déterminer la traduction ekphrasis et une organisation de la description proprement littéraire. Grâce au parallélisme syntaxique asyndétique : «Les tours plus que médiévales / les monstres mécaniques d'acier éclaté » (Rolin, 2019, p. 287), l'auteur souligne son intérêt pour les strates de l'Histoire et les superpositions des temporalités. Il joint ici des tours moyenâgeuses et des chars d'assaut qui ont pris des allures de monstres de contes médiévaux. Il propose une concaténation qui fait se rencontrer différentes époques et fait traverser le palimpseste général du monde auquel il participe. Cet amour pour les traces anciennes se perçoit dans toute sa littérature et notamment dans Paysages originels au sein duquel l'auteur évoque ses recherches pour redécouvrir les lieux et rues où ont vécu ses écrivains adulés. Le paysage dans cet extrait s'organise selon une verticalité et un regard en surplomb très large sur la ville en contrebas qui permet d'envisager à travers cette vision les strates compilées de l'Histoire d'un lieu. Il y inclut des remarques de détail d'une vision rapprochée (sa tourelle éjectée à cinq mètres). Le loin et le près s'unissent dans un même « tableau » qui révèlent une vérité du chaos du monde. Rolin s'établit au bord du monde comme témoin et scribe de pans de paysages qui disent le monde et interrogent la notion de point de vue narratif.

Rolin a la mémoire de vieille " tortue », est une bibliothèque qui colporte la littérature au sens où le définit Auguste Comte en précisant que les morts gouvernent 
les vivants. Il est également le compulsif scribe des traces des villes, des rues, des paysages qu'il porte en lui comme un cartographe d'une représentation du monde dessinée par sa mémoire sélective, faillible et inventive... Il est le géoauto-biographe décentré du récit du monde continué.

L'écrivain s'est engagé à travers l'ensemble de sa littérature dans l'élaboration d'une vaste géographie personnelle qui s'étend sur les territoires traversés depuis une cinquantaine d'années, qui s'ancre également dans l'Histoire en mettant au jour les strates enfouies des ruines de ce qui fut et qui enfin s'articule avec une bibliothèque cosmopolite des grands auteurs des siècles précédents. Par exemple, l'ouvrage L'Invention du monde fut l'occasion pour l'auteur de mettre en œuvre le projet ambitieux de l'extension de sa géographie personnelle à l'échelle de la planète. En effet, il envisage de dire le monde à partir de la lecture croisée des journaux de la planète publiés le même jour. Il désire ainsi, dans un mouvement de circumnavigation scriptural, exprimer la diversité du monde et le colossal bricà-brac qui agite notre planète pour établir une restitution littéraire qui exprime au mieux le monde par le récit. Par conséquent, il assigne à l'écrivain la place de l'archiviste géographe qui recense les êtres, les reliefs, les événements... pour en ressusciter le surgissement à travers les traces et les ruines ou qui prolonge leur existence par la naissance du texte conditionné à des ambitions à la hauteur d'un impossible et à une prédilection pour un projet totalisant. Il invente ainsi un territoire qui lui est propre mais à partir d'une extériorité dont il devient le reflet. Les voyages lui permettent de se déprendre de lui-même et de résonner d'une vibration du monde entier dont il colporte les vicissitudes et dont il est un témoin en ajoutant au monde une strate nouvelle par ses livres.

En définitive, il me semble que ce qui est absolument contemporain pour moi en tant qu'écrivain, je veux dire ce qui constitue le territoire qu'il me revient d'explorer et de mettre en forme littéraire, c'est ce que je dois léguer, ce qui sans moi ne serait pas transmis : ce qui est donc, avec moi, en voie de disparition (Rolin, 2011, p. 66).

Ainsi, Rolin articule un mouvement qui prend appui sur le présent pour organiser un retour sur un espace préexistant qu'il s'attache à la fois à reconnaître physiquement par ses déplacements sur le terrain et à transférer dans une traduction symbolique livresque. Il se veut héritier de paysages, de décombres, de rencontres... dont seule la littérature pourrait assurer une pérennité et dont il ne serait que le relais dans une activation et résurgence continue par son labeur. En réalité, cette tentative de dire le monde à partir de son époque est liée à un mauvais " placement", à une impression de ne pas appartenir à son époque ou d'être de toutes les époques, tel Chateaubriand mal placé dans son siècle ${ }^{4}$. Rolin explique,

4 « Pourquoi suis-je venu à une époque où j'étais si mal placé ?» (Chateaubriand, 1951/1841, p. 923). 
à propos de son goût pour les récits d'un voyage toujours continué et à propos de l'écriture contrainte ${ }^{5}:$ " je crois qu'on écrit parce qu'on est mal placé dans son époque, parce qu'on s'y sent dépaysé » (Rolin, 2008) ${ }^{6}$. Le regard neuf qu'il porte constamment sur les paysages du monde provient de ce décalage, cultivé avec le temps, d'un homme observant le monde avec l'étrangeté très baudelairienne pour en restituer la beauté. Rolin se sent doublement « dépaysé », c'est-à-dire proprement expatrié par le privatif « dé » et exilé de ces paysages habituels en qualité de perpétuel bourlingueur. L'écrivain ajoute dans un autre entretien : « il me semble qu'il y a une espèce de nécessité pour un écrivain à être sans lieu, déplacé, en situation d'exil » (Rolin, 2002). Rolin se définit donc comme un «mal placé » et cette posture nourrit sa littérature. Il est parvenu à s'extraire de ses années d'engagement maoïste ${ }^{7}$ pour gagner un exil constant au monde qui sera la source même de ses écrits. Il aime à se comparer à un Ulysse sans Ithaque. Il peut être défini comme un témoin précieux pour la littérature.

Rolin préfère pour ses voyages et ses découvertes des lieux marqués par une histoire ou des événements qui ont modifié profondément le paysage. Il fait montre d'une attirance pour les ruines modernes ou anciennes qui constituent pour lui des lieux de déchiffrement du monde faisant écho aux ruines du texte, c'est-à-dire aux images et bribes conservées à la suite de la lecture d'un récit, d'un roman... Rolin a engagé une vaste entreprise de déchiffrement de la réalité dans sa diversité, ses superpositions d'époques qui rendent possible la lecture du monde. « Je m'aperçois que les paysages dont j'ai évoqué le pouvoir de fascination sont des paysages de ruines » (Rolin, 2019, p. 290), précise l'auteur, qui aime à proposer de lire plusieurs strates de l'Histoire à la fois qu'il convoque dans un souci de superposition des époques, de collision des contextes et de la succession des hommes. "Les images stéréoscopiques que ma mémoire superpose à celles que me livrent mes yeux me donnent une vision du temps dans sa profondeur » (Rolin, 2019, p. 257), ainsi l'auteur, à l'instar de Marcel Proust à propos du rapport de la littérature au temps et aux fulgurances, livre sa « méthode » de travail qui repose sur le double mouvement de l'homme qui observe (vision diplopique humaine et présence effective de l'homme face à un site) et de la mémoire qui conserve une « ruine» de l'examen du monde. Le transfert dans le récit additionne ces deux mouvements pour cerner une sensation diffuse et puissante du temps, qui permet à l'écrivain de « dire » le temps et l'hébétude vertigineuse qui est liée à cette perception. Si Rolin a cru en l'Histoire dans la mesure où son engagement

5 « sensiblement leur auteur a été contraint »(Bataille, Le Bleu du ciel, 1934). L'écriture contrainte est celle qu'impose le réel à l'écrivain.

6 Propos recueillis par Nathalie Crom. Publié le 22 août 2008.

7 Rolin anime le comité Viêt-Nam, en mai 1968 mène des manifestations, adhère à la Gauche prolétarienne, dans les années 70 participe à des attentats, orchestre des enlèvements, vit clandestinement... 
politique et révolutionnaire des années 70 avait pour objectif d'y participer par une inflexion des destinées des hommes et des pays..., sa croyance en l'Histoire s'est déplacée vers l'observation de l'empilement des époques qui font l'Histoire, passant d'activiste à contemplateur et témoin de présences anciennes.

Que nous reste-t-il d'une lecture d'un roman, d'un récit de voyage...? quelques images fortement inscrites en nous d'une reconstruction mentale en raison de la rencontre avec des passages de l'œuvre qu'on dit particulièrement bien réussis, c'est-à-dire qui ont conservé en eux quelque chose extorqué au réel que l'écriture tente de cerner. Par exemple Rolin, qui cherche à transmettre et à perpétuer une intensité des images du monde et des impressions, précise que le livre s'organise de la même manière dans la mesure où il propose " un monde » dont le lecteur ne conserve que des émotions, reconstructions mentales, images puissantes, des sortes de « ruines » de l'œuvre. Il précise à ce titre : « Lorsque nous avons oublié tours et détours de l'intrigue, ce qui subsiste et continue de rayonner dans la pénombre où toute la machine textuelle est tombée ce sont des visions en quoi se concentre l'énergie du livre » (Rolin, 2011, p. 72 ). Peu importe pour Rolin la tension narrative au sens où la définit Raphaël Baroni, ce qui prévaut pour l'auteur, c'est la virulence et la persistance des images que le livre propose. Ainsi, le livre est constitué comme un ensemble nécessaire dont certaines parties produisent une impression plus forte et qui constitueront la mémoire du livre comme celle constituée face à un paysage. L'auteur fait exister une concomitance féconde ${ }^{8}$. Il affectionne également de mettre en œuvre le "simultané du non simultané » (Hartog, 2013, p. 212) selon l'expression de François Hartog à propos du travail de Rolin, produisant ainsi un trouble certain pour le lecteur. L'écrivainbourlingueur parvient à élaborer par cette approche une géo-auto-graphie de son existence fondée sur la mémoire des lieux véhiculée par l'individu sensible. Il dessine en creux son autobiographie mais également en plein lorsqu'il livre quelques témoignages d'expérience intimes.

Rolin est donc un écrivain qui, par ses périples poursuivis pendant près d'un demi-siècle, tente de se déprendre de soi ; il met en œuvre un décentrement par une confrontation à ce qu'il définit par l'extériorité à soi, c'est-à-dire les paysages et les êtres. L'écrivain-bourlingueur, compose une géo-auto-graphie de sa présence et une trajectoire de vie fondée sur la mémoire des lieux et des hommes. Sa démarche articule écriture de soi et extériorité que Gérard Cartier exprime dans une belle formule : « La littérature est, avec l'Histoire et la Géographie, le troisième pôle du triangle magique qui structure l'imaginaire d'Olivier Rolin » (Cartier, 2017, p. 7). L'auteur s'inscrit dans une filiation littéraire qui va d'Homère à lui en passant par

8 « le génie métonymique du roman consiste en cela aussi qu'il fait voir les grandes choses à travers les petites, qu'il fixe les grandes visions dans l'éclat bref et mémorable d'une image » (Rolin, 2011, p. 52). 
Marcel Proust. Il évoque la puissance de la littérature comme ses prédécesseurs, précise son espace créatif par un propos théorique sur son propre travail et place sa littérature sous un aspect obituaire qui lui permet de gagner la liberté d'un écrivain « désamarré ».

\section{References}

Chateaubriand, R. (1951/1841). Mémoires d'outre-tombe. Paris: La pléiade.

Baroni, R. (2007). La Tension narrative, Paris: Seuil.

Bouvier, N. (1997). Routes et Déroutes. Paris: Métropolis.

Cartier, G. (2017). Invitation en Rolinie. Europe, 1058,1059,-1060, 7.

De Montaigne, M. (1963/1588). Les Essais. Paris: La pléiade.

Hartog, F. (2013). Croire en l'histoire. Paris: Flammarion.

Proust, M. (1925). Albertine disparue. Paris: Gallimard.

Rolin, O. (1993). L'Invention du monde. Paris: Seuil.

Rolin, O. (2002). Tauromachie avec les mots, entretien Yves Charnet. Retrieved June 5, 2020, from https://remue.net/Olivier-Rolin-Tauromachie-avec-les-mots.

Rolin, O. (2008). La littérature m'a appris l'ambiguïté. Télérama 3058. Retrieved June 5, 2020, from https://www.telerama.fr/livre/olivier-rolin-la-litterature-m-a-appris-l-ambiguite,32601.php.

Rolin, O. (2011). Bric et broc. Paris: Verdier.

Rolin, O. (2019). Extérieur monde. Paris: Gallimard. 